Review

\title{
What Is the Mechanism Driving the Reduction of Cardiovascular Events from Glucagon-like Peptide-1 Receptor Agonists?-A Mini Review
}

\author{
Jared Berndt ${ }^{1,2}$, Soo Liang Ooi ${ }^{1}$ (D) and Sok Cheon Pak ${ }^{1, *(\mathbb{D}}$ \\ 1 School of Dentistry and Medical Sciences, Charles Sturt University, Bathurst, NSW 2795, Australia; \\ jared.berndt@lilly.com (J.B.); sooi@csu.edu.au (S.L.O.) \\ 2 Eli Lilly Australia Pty. Ltd., West Ryde, NSW 2114, Australia \\ * Correspondence: spak@csu.edu.au; Tel.: +61-2-6338-4952; Fax: +61-2-6338-4993
}

check for

updates

Citation: Berndt, J.; Ooi, S.L.; Pak, S.C. What Is the Mechanism Driving the Reduction of Cardiovascular Events from Glucagon-like Peptide-1 Receptor Agonists?-A Mini Review. Molecules 2021, 26, 4822. https:// doi.org/10.3390/molecules26164822

Received: 5 July 2021

Accepted: 6 August 2021

Published: 10 August 2021

Publisher's Note: MDPI stays neutral with regard to jurisdictional claims in published maps and institutional affiliations.

Copyright: (c) 2021 by the authors. Licensee MDPI, Basel, Switzerland. This article is an open access article distributed under the terms and conditions of the Creative Commons Attribution (CC BY) license (https:// creativecommons.org/licenses/by/ $4.0 /)$.

\begin{abstract}
Glucagon-like peptide-1 receptor agonists (GLP-1 RAs) are considered the standard of care for type 2 diabetes in many countries worldwide. These molecules have profound antihyperglycaemic actions with a favourable safety profile. They are now being considered for their robust cardiovascular (CV) protective qualities in diabetic patients. Most recent $\mathrm{CV}$ outcome trials have reported that GLP-1 RAs reduce major adverse cardiovascular events (MACE). Furthermore, the GLP-1 RAs seem to target the atherosclerotic CV disease processes preferentially. GLP-1 RAs also improve a wide range of routinely measured surrogate markers associated with CV risk. However, mediation analysis suggests these modest improvements may contribute indirectly to the overall anti-atherogenic profile of the molecules but fall short in accounting for the significant reduction in MACE. This review explores the body of literature to understand the possible mechanisms that contribute to the $\mathrm{CV}$ protective profile of GLP-1 RAs.
\end{abstract}

Keywords: glucagon-like peptide-1; dipeptidyl peptidase-4; haemoglobin A1c; systolic blood pressure; atherosclerosis; mononuclear cells; vascular smooth muscle cells; mitochondria; endothelial cells

\section{Introduction}

Diabetes is a global medical epidemic growing each year; it is also a major risk factor for cardiovascular disease (CVD) and associated morbidity. Furthermore, macrovascular complications account for most hospitalisations and deaths for patients with diabetes [1]. Glucagon-like peptide-1 (GLP-1) receptor agonists (RAs) are considered the standard of care for treating type 2 diabetes (T2D) by the American Diabetes Association [2]. GLP-1 RAs show a robust and sustained reduction in blood glucose markers with a favourable safety profile. In recent years, they are equally considered for their impressive cardiovascular (CV) protection benefit [3].

GLP-1 RAs are synthetic forms of the native GLP-1 produced in the L cells of the small intestine. Native GLP-1 is released from the gut in response to oral glucose intake, and this is commonly referred to as the incretin effect [4]. Endogenous GLP-1 has a plethora of actions throughout the body, including stimulating glucose-mediated insulin release from pancreatic beta cells, inhibiting glucagon secretions from pancreatic alpha cells, slowing gastric emptying, and promoting satiety signals in the central nervous system [5]. Native GLP-1 is rapidly degraded by the enzyme dipeptidyl peptidase-4 (DPP-4) in physiologic conditions. GLP-1 RAs are engineered to be resistant to DPP-4, thus they can augment the incretin effect [6]. GLP-1 receptors (GLP-1Rs) are widely distributed throughout the CV system, and subsequently, GLP-1 RAs have been shown in large cardiovascular outcome trials (CVOTs) to elicit CV protection, irrespective of their glucose-lowering ability [7].

GLP-1 RAs have been prescribed for primary and secondary prevention of CVD [2]. However, the primary mechanism that is driving CV protection remains enigmatic. Table 1 
shows a list of CVOTs reporting the MACE reduction effects of different GLP-1 RA molecules compared to the placebo. A recent meta-analysis confirms that the overall class of GLP-1 RAs significantly reduced major adverse cardiovascular events (MACE) by $12 \%$ (Hazard ratio $=0.88,95 \%$ confidence interval: $0.84-0.94 ; p<0.001$ ) [3]. Efpeglenatide was not included in this meta-analysis as its results were published later. As shown in Table 1, five out of seven CVOTs reported significant reductions of MACE versus placebo, whereas two of the studies reported neutral results. Interestingly, both GLP-1 RAs that produced a neutral effect had a daily dosing regimen instead of weekly $[8,9]$.

Table 1. The effects of GLP-1 RAs on relative MACE reduction compared to placebo: The results from CVOTs.

\begin{tabular}{llccc}
\hline Author (Year) & Molecule & N & Hazard Ratio (95\% CI) & $p$-Value \\
\hline Pfeffer et al. (2015) [8] & Lixisenatide & 6068 & $1.02(0.89-1.17)$ & 0.81 \\
Marso et al. (2016) [10] & Semaglutide & 3297 & $0.74(0.58-0.95)$ & $0.02^{*}$ \\
Holman et al. (2017) [9] & Exenatide & 14,752 & $0.91(0.83-1.00)$ & 0.06 \\
Hernandez et al. (2018) [11] & Albiglutide & 9463 & $0.78(0.68-0.90)$ & $0.0006^{*}$ \\
Zinman et al. (2018) [12] & Liraglutide & 9340 & $0.87(0.78-0.97)$ & $0.01^{*}$ \\
Gerstein et al. (2019) [7] & Dulaglutide & 9901 & $0.88(0.79-0.99)$ & $0.026^{*}$ \\
Gerstein et al. (2021) [13] & Efpeglenatide & 4076 & $0.73(0.58-0.92)$ & $0.007^{*}$ \\
\hline
\end{tabular}

Abbreviation: CI, confidence interval; CVOTs, cardiovascular outcome trials; MACE, major adverse cardiovascular events; $\mathrm{N}$, number of participants; ${ }^{*}$ Significant results with $p<0.05$.

Another GLP-1 RA, taspoglutide, is not mentioned in Table 1 as it was never commercialised due to tolerability issues that significantly disrupted the associated CVOT. Comparable data are thus unavailable [14]. Nevertheless, a meta-analysis combining all available randomised control trials on taspoglutide has reported its CV safety [15]. One more incretin mimetic of interest is tirzepatide. This novel dual GLP-1Rs/glucosedependent insulinotropic polypeptide co-agonist has the most promising effect on weight loss and haemoglobin A1c (HbA1c) to date [16]. Tirzepatide is currently being investigated in a large CVOT, with results expected in the coming years [17].

The MACE components that show the most prominent trend in reduction appear to be associated with atherosclerotic pathways [3]. The finding suggests that mechanistic analysis may detect anti-atherogenic properties of GLP-1 RAs throughout the vascular tissue. Additionally, GLP-1Rs are ubiquitously expressed throughout the cells that are involved in atherosclerosis pathogenesis [18]. The results of these CVOTs pose further research questions as to what predominant mechanism is driving the $\mathrm{CV}$ protection from these molecules. The CV surrogate markers measured in these studies are not able to fully account for the extensive CV benefit produced; hence, there is an identifiable gap in the literature. Therefore, there is a need to understand the cause and effect of GLP-1 RAs on $\mathrm{CV}$ benefit within the relevant $\mathrm{CV}$ tissues.

The interaction of GLP-1 RAs with the GLP-1Rs and the subsequent intracellular pathways are well defined in tissues outside the CV system [19]. Previous reviews have looked at both endogenous and exogenous incretins [6] and DPP-4 inhibitors [20] in the context of CVD. Additionally, a more recent review has a similar research question and includes a comparable array of GLP-1 RAs. However, it has a wider focus on both diabetic kidney disease and CVD [21]. This mini-review differs from the previous research in that it is addressing exclusively injectable synthetic GLP-1 RAs' effect on CVD as opposed to endogenous GLP-1. In addition, this paper focuses primarily on atherosclerotic CVD consistent with the data in CVOTs.

As shown in Figure 1, this review will first look at the possible indirect effects of the GLP-1 RAs on CVD throughout the entire body. The review will also analyse the effect of incretin mimetics on well-understood CVD surrogate markers, including blood glucose, blood pressure (BP), lipids and weight. The paper will then explore the direct relationship between GLP-1 RAs and atherosclerosis pathogenesis by systematically separating the 
pre-clinical studies into structural components of the vasculature to help the readers conceptualise how the molecules affect different tissues and interact with different cells.

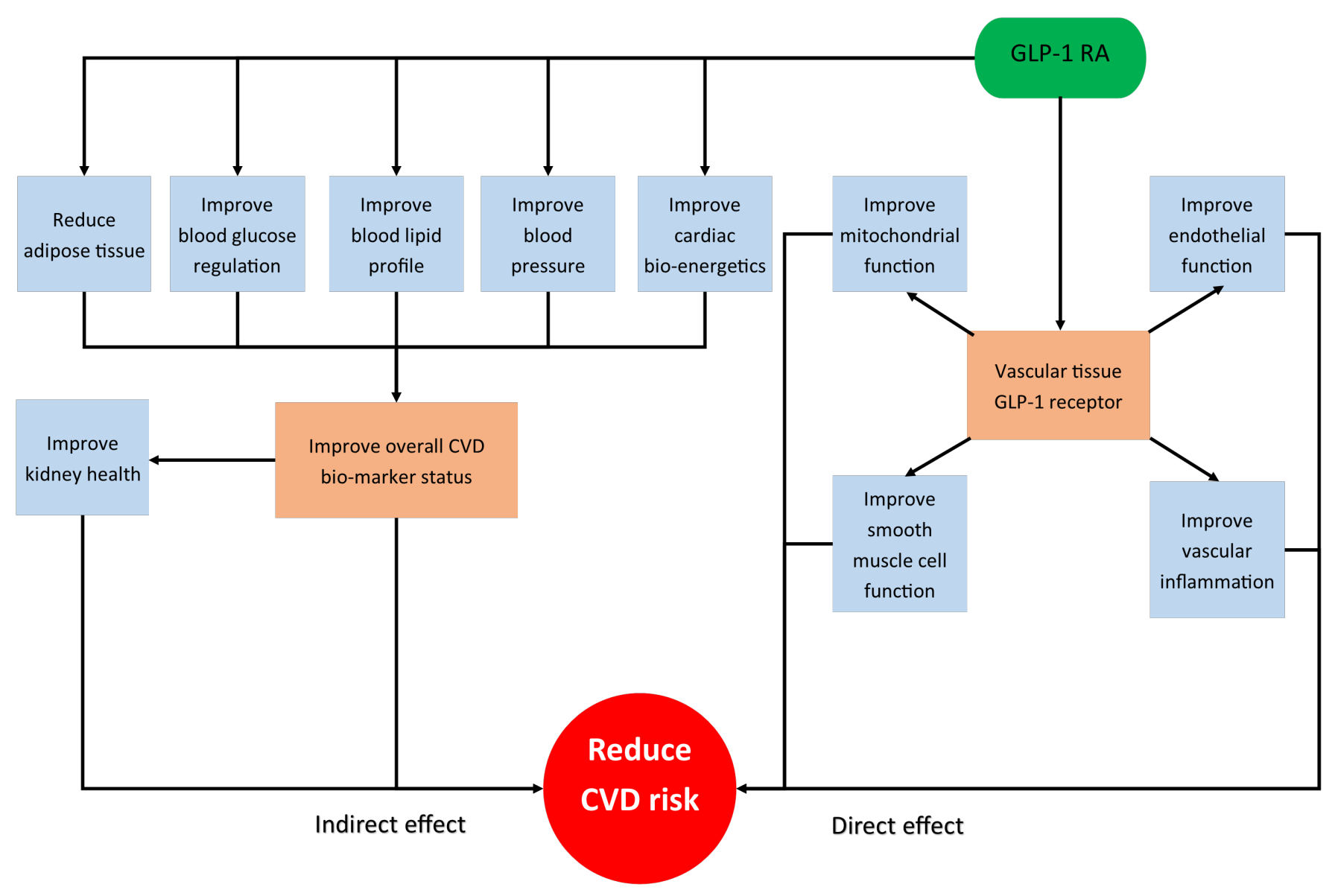

Abbreviations: CVD, cardiovascular disease; GLP-1, glucagon-like peptide-1; RA, receptor agonist.

Figure 1. A schematic that outlines the direct and indirect actions of GLP-1 RAs in reducing CVD risk.

\section{Indirect Effects of GLP-1 RAs on CVD Risk Reduction}

\subsection{Kidneys}

Kidney outcomes in all the included GLP-1 RA CVOTs are secondary endpoints and therefore are exploratory without the statistical power to claim any specific renal benefit. A pooled analysis across all the CVOTs found that the composite kidney outcome reduced by $17 \%$ compared to the placebo, and the largest data signal was for a reduction in albuminuria [22]. However, the variances of reno-protection between molecules could be attributed to differences in trial design [23]. In a recent review, van Baar et al. [24] suggest that GLP-1 RAs can protect kidney health directly or indirectly. GLP-1 RAs have been shown to reduce blood glucose and modestly improve BP, adiposity and serum lipids, which, when aggregated, can indirectly affect renal health. Moreover, the kidneys widely express the GLP-1Rs, although their exact location within the tissue remains unknown [25].

A recent review summarises the body of literature describing primarily natriuretic and an immunomodulatory effect of GLP-1 RAs, causing the reno-protective effect [26]. The consistent data trends seen across the body of literature has validated the need for further analysis. Currently, there is an ongoing clinical trial with semgalutide exploring its effect in patients with chronic kidney disease. This study will be the first large scale GLP-1 RA study with kidney outcomes forming the primary endpoint [27]. 


\subsection{Blood Glucose, BP, Lipids and Weight}

GLP-1 RAs significantly reduce $\mathrm{HbA1c}$ compared to the placebo, and as reported in a mediation analysis of dulaglutide, $\mathrm{HbA} 1 \mathrm{c}$ only mediates a modest proportion of the overall $\mathrm{CV}$ benefit $[3,28]$. Glucose-lowering does decrease $\mathrm{CV}$ risk; however, the method of lowering blood glucose matters. GLP-1 RAs lower the blood glucose without additional CVD burden. In contrast, other anti-hyperglycaemic molecules may counteract the $\mathrm{CV}$ protective effect of glucose-lowering through harmful off-target mechanisms [29,30]. Out of the CVOTs, semaglutide has shown the most significant ability to reduce weight [10]. Additionally, weight loss driven by GLP-1 RAs is likely to influence the overall CV protective profile of the molecules positively $[10,31,32]$. However, most molecules available fall short of clinically significant weight loss that would sufficiently account for the overall CV protection [33]. On the contrary, subgroup analysis of dulaglutide by Gerstein et al. [7] has shown that the CV benefit from GLP-1 RAs occurs irrespective of weight loss.

The CVOTs report modest improvement in the lipid profile from GLP-1 RAs treatment. However, there was a significant proportion of patients in these studies receiving lipid modulating therapy [3,7,22]. A recent systemic review reported that GLP-1 RAs can modestly alter postprandial serum lipids via the alteration of hepatic triglyceride output [34]. Furthermore, the review proposed additional mechanisms that improve lipid metabolisms, such as improved peripheral bio-energetics and increased endogenous insulin functionality [34]. Despite most patients in the large CVOTs being on the standard hypertension care, GLP-1 RAs can modestly augment systolic BP reduction [3]. The mechanism has been investigated in vitro whereby liraglutide caused an increase in vasodilation in the arterial vasculature and decreased vascular contraction, causing a reduction in systolic BP [35]. Notwithstanding, a mediation analysis of dulaglutide reports that BP reduction does not significantly contribute to the overall CV protection [28].

\subsection{Myocardium}

The body of literature suggests that GLP-1 RAs directly affect the myocardial tissue and indirectly change the peripheral circulating energy substrates [36]. A recent systematic review cited limited expression of GLP-1Rs in the ventricular myocardium, suggesting the increase in cardiac efficiency must be a consequence of GLP-1Rs stimulation in the coronary vascular smooth muscle cells [37]. Moreover, the remainder of the protective benefit must be derived from the peripheral vasculature with a high expression of GLP1Rs [37]. Another possible mechanism in which GLP-1 RAs can influence the myocardial tissue is through bioenergetics. The myocardium has metabolic flexibility using glucose and fat substrates for adenosine triphosphate production in a healthy person. In the context of T2D, there is a marked increase in circulating fatty acids. Subsequently, the predominant substrate for myocardial cells becomes fatty acids instead of having a mixture of fuels [36].

Improved blood glucose control may also enhance the availability to use alternate substrates, such as ketones and lactate, for fuel. This 'thrifty substrate' hypothesis has been proposed for the sodium-glucose cotransporter 2 inhibitors, another T2D medication class with a proven CV benefit [38]. Incretin molecules have been shown to potentially reduce the severity of myocardial infarction (MI) by a reduction of infarct size, as reported in pre-clinical studies [39]. Incidentally, GLP-1 RAs have a structural resistance to DPP-4mediated degradation and do not form the same metabolites as endogenous GLP-1. These endogenous metabolites may have their own unique $\mathrm{CV}$ protective mechanisms outside of GLP-1R stimulation [37]. Moreover, a recent review suggests that DPP-4 inhibition may be associated with several anti-atherogenic pathways independent of an increase in endogenous GLP-1 and glucose-dependent insulinotropic polypeptide [20].

\section{Direct Effects of GLP-1 RAs on CVD Risk Reduction}

\subsection{Immune Response}

Atherosclerosis is a condition characterised by chronic inflammation in the vascular walls. The dominant immune cells implicated in the formation of atherosclerotic plaques 
are mononuclear leukocytes. GLP-1 RAs may contribute to modulating the immune response to atherogenic environments, which can, in turn, assist in the stabilisation of existing lesions. Lixisenatide is reported to bind to monocytes' GLP-1Rs and modulate the immune response via increased intracellular 5'-AMP-activated protein kinase (AMPK) [40]. Similar results have been replicated with liraglutide downregulating proinflammatory cytokines via an increase in AMPK [41].

Additionally, through analysing messenger ribonucleic acid expression in GLP-1 RAtreated cells, there are reportedly decreased levels of proinflammatory mediators and their precursors of tumour necrosis factor (TNF)- $\alpha$, monocyte chemoattractant protein- 1 and nuclear factor (NF)- $\kappa \mathrm{B}$ [42]. Moreover, when genetically prone atherosclerotic mice were treated with semaglutide or liraglutide, they had reduced plaque formation as well as attenuated levels of TNF- $\alpha$ and interferon- $\gamma$ [43]. Similar results were replicated in a murine model where liraglutide was able to reduce the total plaque burden and increase the stability of existing plaques. Interestingly, when co-administering a GLP-1R antagonist, the effect of liraglutide on plaques was able to be partly attenuated [44]. This finding suggests that GLP-1 RAs may have immunomodulating effects in the vascular walls and may prevent the development of atherosclerotic plaques in patients that express high $\mathrm{CV}$ risk phenotypes. These results also indicate that the effects of GLP-1 RAs are likely to be driven significantly from GLP-1R activation.

Pro-atherogenic inflammation causes the recruitment of monocytes into the vascular wall. The subsequent transformation of monocytes into macrophages and eventually into foam cells is central to the pathogenesis of atherosclerosis [45]. Cultured monocytes treated with exenatide show an increase in autophagy of chemotactically recruited monocytes [46]. Macrophages seem to have different phenotypes that drive different behaviours. Exenatide has been reported to favourably manipulate the phenotype of the macrophages from M1 to M2 [47].

Furthermore, liraglutide also shifts the proportion of M2 macrophages compared to M1 in mice treated with placebo $[48,49]$. These changes to the macrophage phenotype reduce deleterious changes in the vascular wall and promote the favourable stabilisation of atherosclerotic lesions. Liraglutide-treated mice were able to recruit a more favourable immune infiltrate into atherosclerotic lesions, which reduces overall atherosclerotic burden $[48,49]$. Furthermore, exenatide can attenuate reactive oxygen species (ROS) production from stressed macrophages [50]. Such observation further suggests the ability of GLP-1 RAs in modulating harmful immune responses that drive the formation of plaques.

\subsection{Vascular Smooth Muscle Cells (VSMC)}

VSMC dysfunction can drive pro-atherogenic conditions in the vascular walls, including disrupting vascular tone and increasing the switch from a contractile to a proliferative phenotype. This phenotype change in the VSMC may drive pathogenic remodelling in the context of atherogenic signals [51]. Exenatide has demonstrated the ability to mitigate this dysfunction by modulating the phenotypic switching of VSMC in cell culture [52]. The authors reported that the stimulation of GLP-1R demonstrated an increase in AMPK phosphorylation and an increase in intracellular Sirtuin 1 and Forkhead box O3. Therefore, these pathways are the likely mediators between GLP-1 RAs and the correction of the VSMC function [52].

In an atherosclerotic murine model, liraglutide was able to mitigate the actions of angiotensin II (ANG II) in VSMC, which delayed the progression of plaque formation and promoted the stabilisation of existing lesions [53]. The ability of GLP-1 RAs to increase intracellular AMPK and subsequent downstream consequences on cell cycling may be responsible for the attenuation of pathological remodelling of the vasculature [53]. In addition, cultured aortic VSMC treated with exenatide ameliorate the deleterious effects of ANG II by offsetting extracellular signal-regulated kinase (ERK) and c-Jun N-terminal kinase phosphorylation [54]. These pathways are typically stimulated by ANG II and cause pro-atherogenic remodelling. The ability to mitigate atherogenic signals by GLP-1 RAs 
may also be through the inhibition of the phosphoinositide 3-kinases and protein kinase B pathways [55].

A review explores the important roles of matrix metalloproteinases (MMPs) and tissue inhibitors of metalloproteinases (TIMPs) in atherosclerosis development [56]. Recent preclinical data suggest that GLP-1 RAs may lead to CV protection through the modulation of the ratio of MMPs and TIMPs. In human endothelial cells, exenatide caused suppressed levels of MMPs and an increase in TIMPs [57]. In another in vitro study, exenatide has also been reported to favourably modulate the expression of MMPs and TIMPs in coronary artery smooth muscle cells [58]. The inhibition of the NF- $\kappa \mathrm{B}$ and Akt signalling pathway has been identified as the likely mediator between exenatide and MMPs in the two studies mentioned above, respectively $[57,58]$.

Interestingly, a plausible mechanism of NF- $\kappa$ B inhibition may be through the GLP1 RA stimulation of adipose adiponectin (APN) [59]. Exenatide has been reported to upregulate APN expression in a murine model [60]. Platelet-derived growth factor (PDGF) is an additional candidate for the underlying mechanism of GLP-1 RAs to modulate the development of atherosclerotic plaque. It has been reported that several GLP-1 RAs are able to mitigate PDGF-induced proliferation of VSMC, influencing the local atherosclerosis physiology [37].

\subsection{Mitochondria}

Another possible protective mechanism of GLP-1 RAs against CVD may be through actions in the mitochondria. Liraglutide-treated rats exposed to a known stressor were able to mitigate mitochondrial dysfunction in VSMC [61]. In contrast, their counterparts treated with a GLP-1R antagonist demonstrated an increase in mitochondrial dysfunction. The authors concluded that liraglutide triggers an increase in CV protection via direct binding to VSMC GLP-1Rs and upregulating various intracellular pathways involved in mitochondrial health [61].

GLP-1 RAs may be able to decrease mitochondria-driven apoptosis in CV tissue. Cells treated with exenatide consistently showed decreased caspase-3 activity, a known marker of apoptosis $[62,63]$. In another study, exenatide demonstrated the ability to mitigate the effects of TNF- $\alpha$ in treated cells by attenuating excessive ROS production and upregulating various other pathways that are pertinent for mitochondrial integrity [64]. Moreover, an in vitro model of murine cardiomyocytes stressed by hypoxic conditions reported decreased mitochondrial ROS production in exenatide-treated cells [62]. Additionally, several intracellular signals associated with mitochondrial health and function were upregulated in those cells.

When exenatide was administered to MI-prone mice, it improved CV performance and decreased deleterious remodelling. Furthermore, exenatide also attenuated attenuated ROS production and improved mitochondrial integrity in stressed cardiomyoblasts [63]. A potential mechanism in which GLP-1 RAs can attenuate ROS production in the mitochondria could be the lectin-like oxidised low-density lipoprotein receptor-1 (LOX-1). Liraglutide could function as a mediator between LOX-1 and mitochondrial ROS production, as demonstrated in a recent study. The researchers were able to blunt the protective effects of liraglutide on ROS production by manipulating LOX-1 activity through antibody treatment [65].

\subsection{Endothelium}

GLP-1 RAs may buffer the vascular endothelial cells from deleterious signalling exhibited by oxidised low-density lipoprotein (ox-LDL). In vitro cell culture of human aortic cells infused with ox-LDL and dulaglutide showed reduced inflammatory cell adhesion, a known precursor of atherosclerosis formation. The biochemical analysis of cell signalling proteins suggests that dulaglutide may buffer the effects of ox-LDL on Krüppel-like factor 2 (KLF2) transcription factors [66]. The KLF2 pathway is pertinent to the maintenance of vascular integrity, and GLP-1 RAs attenuate the effect of ox-LDL on this 
pathway. The same effect was observed with liraglutide in another study, which offered further insights into the intracellular signalling pathway [67]. The authors concluded that GLP-1 RA signalling was dependent on ERK 5, as benefits from liraglutide were mitigated with the inhibition of the ERK 5 pathway [67].

GLP-1 RAs have been reported to decrease vascular adhesion molecules in an in vitro model. Mice treated with liraglutide produced correspondingly improved endothelial function [68]. Additionally, liraglutide was able to attenuate the elevation of vascular cell adhesion molecule-1, intercellular adhesion molecule-1 and E-selectin in liraglutidetreated endothelial cells, likely via the activation of calcium/calmodulin-dependent protein kinase I and AMPK. Interestingly, the co-administration of a GLP-1R antagonist attenuated the effect of liraglutide [68]. The finding suggests that the effect of GLP-1 RAs on the endothelium is mediated through GLP-1R activation.

Endothelin-1 (ET-1) is a potent vasoconstrictor, and its upregulation is typically evident in the endothelium of atherosclerotic disease. As such, elevated ET-1 is implicated in a wide range of CVD pathologies. When cells were treated with liraglutide, reduced ET-1 activity was observed, possibly due to the decreased NF- $\kappa$ B activity [69]. The ability of GLP-1 RAs to inhibit NF- $\kappa$ B is consistently reported in the body of literature.

\section{Conclusions}

Meta-analysis of the GLP-1 RA CVOTs suggests a class effect of CV benefit. However, there were reported differences among the molecules with regards to MACE reduction. Some of the inconsistency may be attributed to differences in study design. Incidentally, the two molecules that did not meet their primary endpoint involved daily dosing instead of weekly. Moreover, the reported pre-clinical trials did not represent a balanced proportion of all the GLP-1 RA molecules; therefore, any differences in their molecular structures that may influence the CV benefits in pre-clinical trials are difficult to confirm.

Additionally, the CVOTs used GLP-1 RAs on top of the standard of care; therefore, it must be considered that these molecules may be working synergistically with another CVD pharmacotherapy. Moreover, the study population in these clinical trials was also diabetic. The CV benefit is yet to be demonstrated in patients without T2D. Furthermore, there seem to be differences among GLP-1 RAs in their ability to reduce various surrogate markers of CVD; however, all molecules demonstrated an overall favourable profile compared to the placebo. There are distinct differences in the metabolism of GLP-1 RAs and native GLP-1, both potentially offering unique and overlapping $\mathrm{CV}$ benefits.

GLP-1 RAs seem to elicit CV protection both directly in the vasculature and indirectly in the periphery. However, what remains unclear is the proportion of this $\mathrm{CV}$ protective mechanism that is driven from local GLP-1R stimulation versus the cumulative peripheral effects that may indirectly improve vasculature function. Moreover, several in vitro studies using GLP-1R antagonists as a control suggest that a significant proportion of the effects of GLP-1 RA is a direct consequence of GLP-1R stimulation and subsequent intracellular cascades. Further investigation of the specific intracellular pathways stimulated by GLP-1R activation is needed.

The reported mechanistic studies consistently demonstrated the attenuation of atherosclerosis-associated processes and seemed to promote signals consistent with the stabilisation of existing lesions. Further investigation is needed to properly quantify the proportional influence of suggested mechanisms of GLP-1 RA CV protection. In conclusion, GLP-1 RAs likely have pleiotropic anti-atherogenic effects throughout the CV system and lead to other favourable metabolic changes in the periphery to produce the overall CV benefit.

Author Contributions: Conceptualisation, writing and original draft preparation, J.B.; writing review and editing, S.L.O. and S.C.P.; visualisation, J.B. and S.L.O.; supervision, S.C.P. All authors have read and agreed to the published version of the manuscript.

Funding: This research received no external funding. 
Acknowledgments: J.B. would like acknowledge Amy Coggins for providing emotional and family support.

Conflicts of Interest: J.B. is an employee of Eli Lilly and Company. This company manufactures and markets GLP-1 RAs.

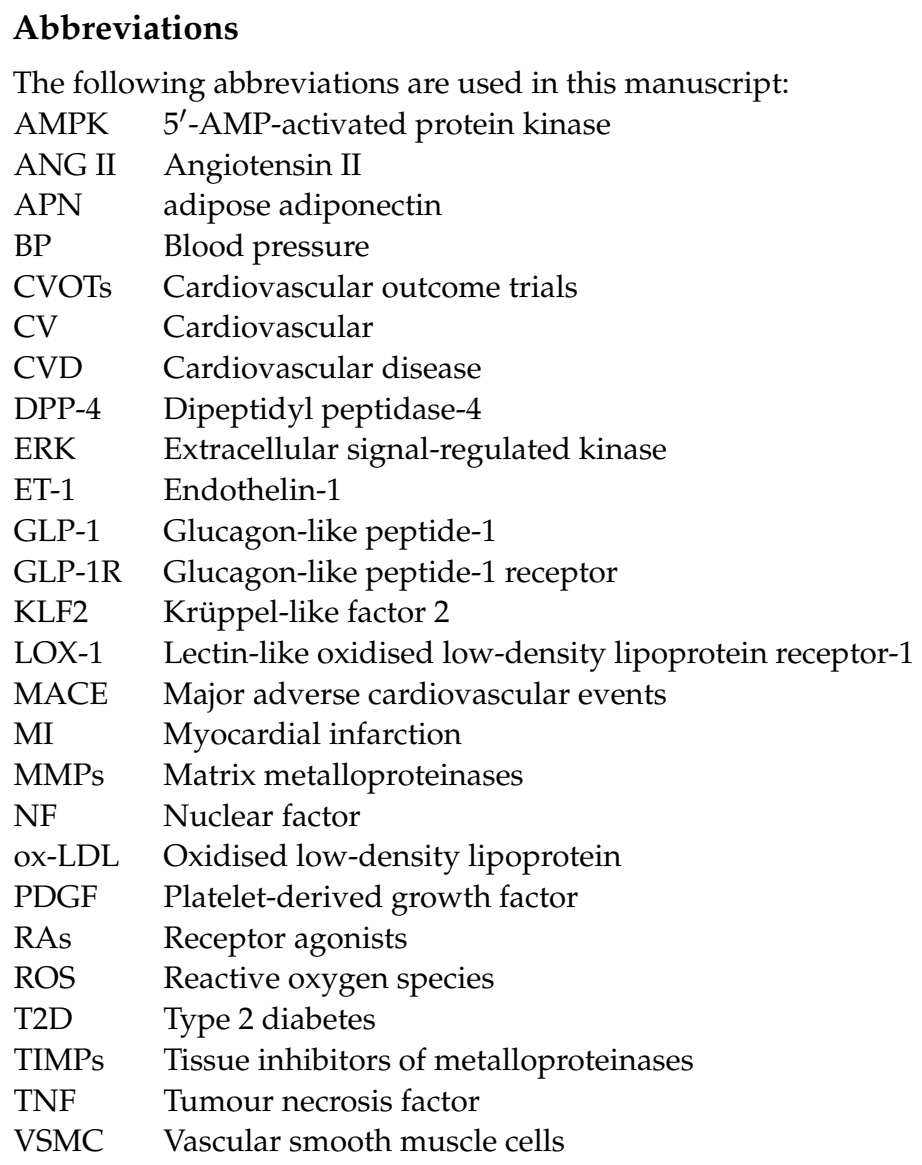

\section{References}

1. Fowler, M.J. Microvascular and macrovascular complications of diabetes. Clin. Diabetes 2008, 26, 77-82. [CrossRef]

2. American Diabetes Association. 9. Pharmacologic approaches to glycemic treatment: Standards of medical care in diabetes-2020. Diabetes Care 2020, 43, S98-S110. [CrossRef]

3. Zelniker, T.A.; Wiviott, S.D.; Raz, I.; Im, K.; Goodrich, E.L.; Furtado, R.H.M.; Bonaca, M.P.; Mosenzon, O.; Kato, E.T.; Cahn, A.; et al. Comparison of the effects of glucagon-like peptide receptor agonists and sodium-glucose cotransporter 2 inhibitors for prevention of major adverse cardiovascular and renal outcomes in type 2 diabetes mellitus. Circulation 2019, 139, $2022-2031$. [CrossRef]

4. Nauck, M.A.; Meier, J.J. Incretin hormones: Their role in health and disease. Diabetes Obes. Metab. 2018,20 (Suppl. 1), 5-21. [CrossRef]

5. Sharma, D.; Verma, S.; Vaidya, S.; Kalia, K.; Tiwari, V. Recent updates on GLP-1 agonists: Current advancements \& challenges. Biomed. Pharmacother. 2018, 108, 952-962. [CrossRef]

6. Drucker, D.J.; Nauck, M.A. The incretin system: Glucagon-like peptide-1 receptor agonists and dipeptidyl peptidase-4 inhibitors in type 2 diabetes. Lancet 2006, 368, 1696-1705. [CrossRef]

7. Gerstein, H.C.; Colhoun, H.M.; Dagenais, G.R.; Diaz, R.; Lakshmanan, M.; Pais, P.; Probstfield, J.; Riesmeyer, J.S.; Riddle, M.C.; Rydén, L.; et al. Dulaglutide and cardiovascular outcomes in type 2 diabetes (REWIND): A double-blind, randomised placebo-controlled trial. Lancet 2019, 394, 121-130. [CrossRef]

8. Pfeffer, M.A.; Claggett, B.; Diaz, R.; Dickstein, K.; Gerstein, H.C.; Køber, L.V.; Lawson, F.C.; Ping, L.; Wei, X.; Lewis, E.F.; et al. Lixisenatide in patients with type 2 diabetes and acute coronary syndrome. N. Engl. J. Med. 2015, 373, 2247-2257. [CrossRef] [PubMed]

9. Holman, R.R.; Bethel, M.A.; Mentz, R.J.; Thompson, V.P.; Lokhnygina, Y.; Buse, J.B.; Chan, J.C.; Choi, J.; Gustavson, S.M.; Iqbal, N.; et al. Effects of once-weekly exenatide on cardiovascular outcomes in type 2 diabetes. N. Engl. J. Med. 2017, 377, 1228-1239. [CrossRef] [PubMed] 
10. Marso, S.P.; Bain, S.C.; Consoli, A.; Eliaschewitz, F.G.; Jódar, E.; Leiter, L.A.; Lingvay, I.; Rosenstock, J.; Seufert, J.; Warren, M.L.; et al. Semaglutide and cardiovascular outcomes in patients with type 2 diabetes. N. Engl. J. Med. 2016, 375, 1834-1844. [CrossRef]

11. Hernandez, A.F.; Green, J.B.; Janmohamed, S.; D'Agostino, R.B., Sr.; Granger, C.B.; Jones, N.P.; Leiter, L.A.; Rosenberg, A.E.; Sigmon, K.N.; Somerville, M.C.; et al. Albiglutide and cardiovascular outcomes in patients with type 2 diabetes and cardiovascular disease (Harmony Outcomes): A double-blind, randomised placebo-controlled trial. Lancet 2018, 392, 1519-1529. [CrossRef]

12. Zinman, B.; Marso, S.P.; Christiansen, E.; Calanna, S.; Rasmussen, S.; Buse, J.B. Hypoglycemia, cardiovascular outcomes, and death: The LEADER experience. Diabetes Care 2018, 41, 1783-1791. [CrossRef]

13. Gerstein, H.C.; Sattar, N.; Rosenstock, J.; Ramasundarahettige, C.; Pratley, R.; Lopes, R.D.; Lam, C.S.P.; Khurmi, N.S.; Heenan, L.; Del Prato, S.; et al. Cardiovascular and renal outcomes with efpeglenatide in type 2 diabetes. N. Engl. J. Med. 2021. [CrossRef] [PubMed]

14. National Library of Medicine (U.S.). A Study of Taspoglutide in Patients with Inadequately Controlled Diabetes Mellitus Type 2 and Cardiovascular Disease. Available online: https://clinicaltrials.gov/ct2/show/record/NCT01018173 (accessed on 2 August 2021).

15. Seshasai, S.R.K.; Bennett, R.L.; Petrie, J.R.; Bengus, M.; Ekman, S.; Dixon, M.; Herz, M.; Buse, J.B.; Ray, K.K. Cardiovascular safety of the glucagon-like peptide-1 receptor agonist taspoglutide in people with type 2 diabetes: An individual participant data meta-analysis of randomized controlled trials. Diabetes. Obes. Metab. 2015, 17, 505-510. [CrossRef]

16. Frías, J.P.; Davies, M.J.; Rosenstock, J.; Pérez Manghi, F.C.; Fernández Landó, L.; Bergman, B.K.; Liu, B.; Cui, X.; Brown, K. Tirzepatide versus semaglutide once weekly in patients with type 2 diabetes. N. Engl. J. Med. 2021, 385, 503-515. [CrossRef]

17. National Library of Medicine (U.S.). A Study of Tirzepatide (LY3298176) Compared with Dulaglutide on Major Cardiovascular Events in Participants With Type 2 Diabetes. Available online: https:/ / clinicaltrials.gov/ct2/show / NCT04255433 (accessed on 2 August 2021).

18. Alonso, N.; Julián, M.T.; Puig-Domingo, M.; Vives-Pi, M. Incretin hormones as immunomodulators of atherosclerosis. Front. Endocrinol. 2012, 3, 112. [CrossRef]

19. Matarese, A.; Gambardella, J.; Lombardi, A.; Wang, X.; Santulli, G. miR-7 regulates GLP-1-mediated insulin release by targeting $\beta$-arrestin 1. Cells 2020, 9, 1621. [CrossRef]

20. Vedantham, S.; Kluever, A.K.; Deindl, E. Is there a chance to promote arteriogenesis by DPP4 inhibitors even in type 2 diabetes? A critical review. Cells 2018, 7, 181. [CrossRef]

21. Cox, E.J.; Alicic, R.Z.; Neumiller, J.J.; Tuttle, K.R. Clinical evidence and proposed mechanisms for cardiovascular and kidney benefits from glucagon-like peptide-1 receptor agonists. US Endocrinol. 2020, 16, 80-87. [CrossRef]

22. Kristensen, S.L.; Rørth, R.; Jhund, P.S.; Docherty, K.F.; Sattar, N.; Preiss, D.; Køber, L.; Petrie, M.C.; McMurray, J.J.V. Cardiovascular, mortality, and kidney outcomes with GLP-1 receptor agonists in patients with type 2 diabetes: A systematic review and meta-analysis of cardiovascular outcome trials. Lancet Diabetes Endocrinol. 2019, 7, 776-785. [CrossRef]

23. Pantalone, K.M.; Munir, K.; Hasenour, C.M.; Atisso, C.M.; Varnado, O.J.; Maldonado, J.M.; Konig, M. Cardiovascular outcomes trials with glucagon-like peptide-1 receptor agonists: A comparison of study designs, populations and results. Diabetes Obes. Metab. 2020, 22, 2209-2226. [CrossRef]

24. van Baar, M.J.B.; van der Aart, A.B.; Hoogenberg, K.; Joles, J.A.; Heerspink, H.J.L.; van Raalte, D.H. The incretin pathway as a therapeutic target in diabetic kidney disease: A clinical focus on GLP-1 receptor agonists. Ther. Adv. Endocrinol. Metab. 2019, 10, 2042018819865398. [CrossRef]

25. Skov, J. Effects of GLP-1 in the kidney. Rev. Endocr. Metab. Disord. 2014, 15, 197-207. [CrossRef] [PubMed]

26. Kawanami, D.; Takashi, Y. GLP-1 Receptor Agonists in Diabetic Kidney Disease: From Clinical Outcomes to Mechanisms. Front. Pharmacol. 2020, 11, 967. [CrossRef]

27. National Library of Medicine (U.S.). A Research Study to See How Semaglutide Works Compared to Placebo in People with Type 2 Diabetes and Chronic Kidney Disease. Available online: https: / clinicaltrials.gov/ct2/show / NCT03819153 (accessed on 2 August 2021).

28. Colhoun, H.M.; Hasenour, C.; Riddle, M.C.; Branch, K.; Konig, M.; Atisso, C.; Lakshmanan, M.; Mody, R.; Gerstein, H.C. 924-p: Exploring potential mediators of the cardiovascular benefit of dulaglutide in REWIND. Diabetes 2020, 69, 924-P. [CrossRef]

29. Turnbull, F.M.; Abraira, C.; Anderson, R.J.; Byington, R.P.; Chalmers, J.P.; Duckworth, W.C.; Evans, G.W.; Gerstein, H.C.; Holman, R.R.; Moritz, T.E.; et al. Intensive glucose control and macrovascular outcomes in type 2 diabetes. Diabetologia 2009, 52, 2288-2298. [CrossRef] [PubMed]

30. Herman, M.E.; O'Keefe, J.H.; Bell, D.S.H.; Schwartz, S.S. Insulin therapy increases cardiovascular risk in type 2 diabetes. Prog. Cardiovasc. Dis. 2017, 60, 422-434. [CrossRef] [PubMed]

31. Shah, M.; Vella, A. Effects of GLP-1 on appetite and weight. Rev. Endocr. Metab. Disord. 2014, 15, 181-187. [CrossRef]

32. Pratley, R.E.; Aroda, V.R.; Lingvay, I.; Lüdemann, J.; Andreassen, C.; Navarria, A.; Viljoen, A. Semaglutide versus dulaglutide once weekly in patients with type 2 diabetes (SUSTAIN 7): A randomised, open-label, phase 3b trial. Lancet. Diabetes Endocrinol. 2018, 6, 275-286. [CrossRef]

33. Brown, J.D.; Buscemi, J.; Milsom, V.; Malcolm, R.; O’Neil, P.M. Effects on cardiovascular risk factors of weight losses limited to 5-10. Transl. Behav. Med. 2016, 6, 339-346. [CrossRef] [PubMed]

34. Tsimihodimos, V.; Elisaf, M. Incretins and lipid metabolism. Curr. Med. Chem. 2018, 25, 2133-2139. [CrossRef] 
35. Liu, L.; Liu, J.; Huang, Y. Protective Effects of Glucagon-like Peptide 1 on Endothelial Function in Hypertension. J. Cardiovasc. Pharmacol. 2015, 65, 399-405. [CrossRef] [PubMed]

36. Al Batran, R.; Almutairi, M.; Ussher, J.R. Glucagon-like peptide-1 receptor mediated control of cardiac energy metabolism. Peptides 2018, 100, 94-100. [CrossRef] [PubMed]

37. Ussher, J.R.; Drucker, D.J. Cardiovascular actions of incretin-based therapies. Circ. Res. 2014, 114, 1788-1803. [CrossRef] [PubMed]

38. Ferrannini, E.; Mark, M.; Mayoux, E. CV protection in the EMPA-REG OUTCOME trial: A “thrifty substrate" hypothesis. Diabetes Care 2016, 39, 1108-1114. [CrossRef]

39. Kato, E.T.; Das, S.R.; McGuire, D.K. Antihyperglycemic therapies and cardiovascular outcomes in patients with type 2 diabetes mellitus: State of the art and future directions. Trends Cardiovasc. Med. 2020, 31, 101-108. [CrossRef]

40. Sudo, M.; Li, Y.; Hiro, T.; Takayama, T.; Mitsumata, M.; Shiomi, M.; Sugitani, M.; Matsumoto, T.; Hao, H.; Hirayama, A. Inhibition of plaque progression and promotion of plaque stability by glucagon-like peptide-1 receptor agonist: Serial in vivo findings from iMap-IVUS in Watanabe heritable hyperlipidemic rabbits. Atherosclerosis 2017, 265, 283-291. [CrossRef]

41. Koshibu, M.; Mori, Y.; Saito, T.; Kushima, H.; Hiromura, M.; Terasaki, M.; Takada, M.; Fukui, T.; Hirano, T. Antiatherogenic effects of liraglutide in hyperglycemic apolipoprotein E-null mice via AMP-activated protein kinase-independent mechanisms. Am. J. Physiol. Endocrinol. Metab. 2019, 316, E895-E907. [CrossRef]

42. Arakawa, M.; Mita, T.; Azuma, K.; Ebato, C.; Goto, H.; Nomiyama, T.; Fujitani, Y.; Hirose, T.; Kawamori, R.; Watada, H. Inhibition of monocyte adhesion to endothelial cells and attenuation of atherosclerotic lesion by a glucagon-like peptide-1 receptor agonist, exendin-4. Diabetes 2010, 59, 1030-1037. [CrossRef] [PubMed]

43. Rakipovski, G.; Rolin, B.; Nøhr, J.; Klewe, I.; Frederiksen, K.S.; Augustin, R.; Hecksher-Sørensen, J.; Ingvorsen, C.; Polex-Wolf, J.; Knudsen, L.B. The GLP-1 analogs liraglutide and semaglutide reduce atherosclerosis in ApoE $(-/-)$ and LDLr(-/-) mice by a mechanism that includes inflammatory pathways. JACC Basic Transl. Sci. 2018, 3, 844-857. [CrossRef]

44. Gaspari, T.; Welungoda, I.; Widdop, R.E.; Simpson, R.W.; Dear, A.E. The GLP-1 receptor agonist liraglutide inhibits progression of vascular disease via effects on atherogenesis, plaque stability and endothelial function in an ApoE(-/-) mouse model. Diabetes Vasc. Dis. Res. 2013, 10, 353-360. [CrossRef]

45. Chistiakov, D.A.; Melnichenko, A.A.; Myasoedova, V.A.; Grechko, A.V.; Orekhov, A.N. Mechanisms of foam cell formation in atherosclerosis. J. Mol. Med. 2017, 95, 1153-1165. [CrossRef] [PubMed]

46. Tanaka, M.; Matsuo, Y.; Yamakage, H.; Masuda, S.; Terada, Y.; Muranaka, K.; Wada, H.; Hasegawa, K.; Shimatsu, A.; SatohAsahara, N. Differential effects of GLP-1 receptor agonist on foam cell formation in monocytes between non-obese and obese subjects. Metabolism 2016, 65, 1-11. [CrossRef] [PubMed]

47. Shiraishi, D.; Fujiwara, Y.; Komohara, Y.; Mizuta, H.; Takeya, M. Glucagon-like peptide-1 (GLP-1) induces M2 polarization of human macrophages via STAT3 activation. Biochem. Biophys. Res. Commun. 2012, 425, 304-308. [CrossRef] [PubMed]

48. Bruen, R.; Curley, S.; Kajani, S.; Crean, D.; O’Reilly, M.E.; Lucitt, M.B.; Godson, C.G.; McGillicuddy, F.C.; Belton, O. Liraglutide dictates macrophage phenotype in apolipoprotein E null mice during early atherosclerosis. Cardiovasc. Diabetol. 2017, 16, 143. [CrossRef]

49. Bruen, R.; Curley, S.; Kajani, S.; Lynch, G.; O’Reilly, M.E.; Dillon, E.T.; Brennan, E.P.; Barry, M.; Sheehan, S.; McGillicuddy, F.C.; et al. Liraglutide attenuates preestablished atherosclerosis in apolipoprotein e-deficient mice via regulation of immune cell phenotypes and proinflammatory mediators. J. Pharmacol. Exp. Ther. 2019, 370, 447-458. [CrossRef]

50. Bułdak, Ł.; Łabuzek, K.; Bułdak, R.J.; Machnik, G.; Bołdys, A.; Okopień, B. Exenatide (a GLP-1 agonist) improves the antioxidative potential of in vitro cultured human monocytes/macrophages. Naunyn. Schmiedebergs. Arch. Pharmacol. 2015, 388, 905-919. [CrossRef]

51. Zhang, Y.N.; Xie, B.D.; Sun, L.; Chen, W.; Jiang, S.L.; Liu, W.; Bian, F.; Tian, H.; Li, R.K. Phenotypic switching of vascular smooth muscle cells in the 'normal region' of aorta from atherosclerosis patients is regulated by miR-145. J. Cell. Mol. Med. 2016, 20, 1049-1061. [CrossRef]

52. Liu, Z.; Zhang, M.; Zhou, T.; Shen, Q.; Qin, X. Exendin-4 promotes the vascular smooth muscle cell re-differentiation through AMPK/SIRT1/FOXO3a signaling pathways. Atherosclerosis 2018, 276, 58-66. [CrossRef]

53. Jojima, T.; Uchida, K.; Akimoto, K.; Tomotsune, T.; Yanagi, K.; Iijima, T.; Suzuki, K.; Kasai, K.; Aso, Y. Liraglutide, a GLP-1 receptor agonist, inhibits vascular smooth muscle cell proliferation by enhancing AMP-activated protein kinase and cell cycle regulation, and delays atherosclerosis in ApoE deficient mice. Atherosclerosis 2017, 261, 44-51. [CrossRef]

54. Nagayama, K.; Kyotani, Y.; Zhao, J.; Ito, S.; Ozawa, K.; Bolstad, F.A.; Yoshizumi, M. Exendin-4 prevents vascular smooth muscle cell proliferation and migration by angiotensin ii via the inhibition of ERK1/2 and JNK signaling pathways. PLoS ONE 2015, 10, e0137960. [CrossRef] [PubMed]

55. Shi, L.; Ji, Y.; Jiang, X.; Zhou, L.; Xu, Y.; Li, Y.; Jiang, W.; Meng, P.; Liu, X. Liraglutide attenuates high glucose-induced abnormal cell migration, proliferation, and apoptosis of vascular smooth muscle cells by activating the GLP-1 receptor, and inhibiting ERK1/2 and PI3K/Akt signaling pathways. Cardiovasc. Diabetol. 2015, 14, 18. [CrossRef]

56. Amin, M.; Pushpakumar, S.; Muradashvili, N.; Kundu, S.; Tyagi, S.C.; Sen, U. Regulation and involvement of matrix metalloproteinases in vascular diseases. Front. Biosci. 2016, 21, 89-118. [CrossRef] 
57. Garczorz, W.; Gallego-Colon, E.; Kosowska, A.; Kłych-Ratuszny, A.; Woźniak, M.; Marcol, W.; Niesner, K.J.; Francuz, T. Exenatide exhibits anti-inflammatory properties and modulates endothelial response to tumor necrosis factor $\alpha$-mediated activation. Cardiovasc. Ther. 2018, 36, e12317. [CrossRef]

58. Gallego-Colon, E.; Klych-Ratuszny, A.; Kosowska, A.; Garczorz, W.; Aghdam, M.R.F.; Wozniak, M.; Francuz, T. Exenatide modulates metalloproteinase expression in human cardiac smooth muscle cells via the inhibition of Akt signaling pathway. Pharmacol. Rep. 2018, 70, 178-183. [CrossRef]

59. Wang, X.; Chen, Q.; Pu, H.; Wei, Q.; Duan, M.; Zhang, C.; Jiang, T.; Shou, X.; Zhang, J.; Yang, Y. Adiponectin improves NF- $\kappa$ B-mediated inflammation and abates atherosclerosis progression in apolipoprotein E-deficient mice. Lipids Health Dis. 2016, 15, 33. [CrossRef] [PubMed]

60. Yang, G.; Lei, Y.; Inoue, A.; Piao, L.; Hu, L.; Jiang, H.; Sasaki, T.; Wu, H.; Xu, W.; Yu, C.; et al. Exenatide mitigated diet-induced vascular aging and atherosclerotic plaque growth in ApoE-deficient mice under chronic stress. Atherosclerosis 2017, 264, 1-10. [CrossRef] [PubMed]

61. Wu, Y.C.; Wang, W.T.; Lee, S.S.; Kuo, Y.R.; Wang, Y.C.; Yen, S.J.; Lee, M.Y.; Yeh, J.L. Glucagon-like peptide-1 receptor agonist attenuates autophagy to ameliorate pulmonary arterial hypertension through Drp1/NOX- and Atg-5/Atg-7/Beclin-1/Lc3 $\beta$ pathways. Int. J. Mol. Sci. 2019, 20, 3435. [CrossRef] [PubMed]

62. Chang, G.; Zhang, D.; Liu, J.; Zhang, P.; Ye, L.; Lu, K.; Duan, Q.; Zheng, A.; Qin, S. Exenatide protects against hypoxia/reoxygenation-induced apoptosis by improving mitochondrial function in H9c2 cells. Exp. Biol. Med. 2014, 239, 414-422. [CrossRef]

63. DeNicola, M.; Du, J.; Wang, Z.; Yano, N.; Zhang, L.; Wang, Y.; Qin, G.; Zhuang, S.; Zhao, T.C. Stimulation of glucagon-like peptide1 receptor through exendin-4 preserves myocardial performance and prevents cardiac remodeling in infarcted myocardium. Am. J. Physiol. Endocrinol. Metab. 2014, 307, E630-E643. [CrossRef]

64. Cao, Y.Y.; Chen, Z.W.; Gao, Y.H.; Wang, X.X.; Ma, J.Y.; Chang, S.F.; Qian, J.Y.; Ge, J.B. Exenatide reduces tumor necrosis factor- $\alpha$-induced apoptosis in cardiomyocytes by alleviating mitochondrial dysfunction. Chin. Med. J. 2015, 128, 3211-3218. [CrossRef]

65. Dai, Y.; Mercanti, F.; Dai, D.; Wang, X.; Ding, Z.; Pothineni, N.V.; Mehta, J.L. LOX-1, a bridge between GLP-1R and mitochondrial ROS generation in human vascular smooth muscle cells. Biochem. Biophys. Res. Commun. 2013, 437, 62-66. [CrossRef] [PubMed]

66. Chang, W.; Zhu, F.; Zheng, H.; Zhou, Z.; Miao, P.; Zhao, L.; Mao, Z. Glucagon-like peptide-1 receptor agonist dulaglutide prevents ox-LDL-induced adhesion of monocytes to human endothelial cells: An implication in the treatment of atherosclerosis. Mol. Immunol. 2019, 116, 73-79. [CrossRef] [PubMed]

67. Yue, W.; Li, Y.; Ou, D.; Yang, Q. The GLP-1 receptor agonist liraglutide protects against oxidized LDL-induced endothelial inflammation and dysfunction via KLF2. IUBMB Life 2019, 71, 1347-1354. [CrossRef]

68. Gaspari, T.; Liu, H.; Welungoda, I.; Hu, Y.; Widdop, R.E.; Knudsen, L.B.; Simpson, R.W.; Dear, A.E. A GLP-1 receptor agonist liraglutide inhibits endothelial cell dysfunction and vascular adhesion molecule expression in an ApoE-/ - mouse model. Diabetes Vasc. Dis. Res. 2011, 8, 117-124. [CrossRef] [PubMed]

69. Dai, Y.; Mehta, J.L.; Chen, M. Glucagon-like peptide-1 receptor agonist liraglutide inhibits endothelin-1 in endothelial cell by repressing nuclear factor-kappa b activation. Cardiovasc. Drugs Ther. 2013, 27, 371-380. [CrossRef] 